\title{
Low Doses of Pristine and Oxidized Single-Wall Carbon Nanotubes Affect Mammalian Embryonic Development
}

\author{
Antonio Pietroiusti, ${ }^{\dagger}$ Micol Massimiani, ${ }^{\ddagger}$ Ivana Fenoglio, ${ }^{\S}$ Massimiliano Colonna, ${ }^{\S}$ Federica Valentini, ${ }^{\perp}$ \\ Giuseppe Palleschi, ${ }^{\perp}$ Antonella Camaioni, ${ }^{\star}$ Andrea Magrini, ${ }^{\dagger}$ Gregorio Siracusa, ${ }^{\ddagger}$ Antonio Bergamaschi, \\ Alessandro Sgambato, ${ }^{\#}$ and Luisa Campagnolo ${ }^{\ddagger * *}$

\begin{abstract}
'Department of Biopathology and ${ }^{\ddagger}$ Department of Public Health and Cell Biology, University of Rome "Tor Vergata", Via Montpellier 1, 00133 Rome, Italy, SDepartment of Inorganic, Physical and Material Chemistry and Interdepartmental Center for Studies on Asbestos and other Toxic Particulates "G. Scansetti" and Interdepartmental Center for Nanostructured Interfaces and Surfaces, University of Torino, Via P. Giuria 7, 10125 Turin, Italy, ${ }^{\perp}$ Department of Chemistry, University of Rome "Tor Vergata", Via della Ricerca Scientifica 1, 00173 Rome, Italy, and "Institute of Occupational Medicine and " Istitute of General Pathology, Università
\end{abstract} \\ Cattolica del Sacro Cuore, Largo San Vito 1, 00168 Rome, Italy
}

O ur results suggest that low-dose SWCNTs may induce fetal malformations, that such an effect is exacerbated by oxidation, and that embryotoxicity of SWCNTs can be reliably predicted by the EST.

In the past few years, carbon nanotubes (CNTs), a class of engineered nanomaterials (ENMs) with versatile physicochemical properties, have attracted much interest for a wide variety of applications, from industry to biomedicine, ${ }^{1-3}$ and it can be easily predicted that in the near future many people will come in contact with such material both in the workplace and in the environment. Although the possible consequences of CNT exposure on human health are largely unknown, harmful effects cannot be excluded. In fact, some characteristics of ENMs, like their high surface to volume ratio, may make them potentially more toxic than the micrometric counterpart. ${ }^{4}$ In addition, in comparison with other ENMs, intrinsic properties of CNTs might increase their toxicity; the fiber-like shape and low solubility might indeed account for their high biopersistence, ${ }^{5}$ possibly exerting long-term adverse effects even after sporadic exposure. Furthermore, differences in the presence of contaminants retained during CNTs synthesis, ${ }^{6,7}$ the deliberate introduction of chemical groups (functionalization), ${ }^{8}$ the length, ${ }^{9,10}$ or the presence of defects ${ }^{11-13}$ may also influence their toxicity profile. ${ }^{14}$

Although several in vivo and in vitro studies on the toxicity of CNTs have been performed in the past few years, ${ }^{15,16}$ a comprehensive knowledge of their effects
ABSTRACT Several in vitro and in vivo studies suggest local and systemic effects following exposure to carbon nanotubes. No data are available, however, on their possible embryotoxicity in mammals. In this study, we tested the effect of pristine and oxidized single-wall carbon nanotubes (SWCNTs) on the development of the mouse embryo. To this end, SWCNTs (from $10 \mathrm{ng}$ to $30 \mu \mathrm{g}$ / mouse) were administered to female mice soon after implantation (postcoital day 5.5); 10 days later, animals were sacrificed, and uteri, placentas, and fetuses examined. A high percentage of early miscarriages and fetal malformations was observed in females exposed to oxidized SWCNTs, while lower percentages were found in animals exposed to the pristine material. The lowest effective dose was $100 \mathrm{ng} /$ mouse. Extensive vascular lesions and increased production of reactive oxygen species (ROS) were detected in placentas of malformed but not of normally developed fetuses. Increased ROS levels were likewise detected in malformed fetuses. No increased ROS production or evident morphological alterations were observed in maternal tissues. No fetal and placental abnormalities were ever observed in control animals. In parallel, SWCNT embryotoxicity was evaluated using the embryonic stem cell test (EST), a validated in vitro assay developed for predicting embryotoxicity of soluble chemical compounds, but never applied in full to nanoparticles. The EST predicted the in vivo data, identifying oxidized SWCNTs as the more toxic compound.

KEYWORDS: engineered nanoparticles $\cdot$ carbon nanotubes $\cdot$ embryonic development $\cdot$ embryotoxicity $\cdot$ teratogen $\cdot$ embryonic stem cell test $\cdot$ embryonic stem cells

is still far from being obtained. This gap is even larger concerning their effects on embryonic development, for which only sparse data are available, most of them referring to aquatic species. ${ }^{17-19}$ Many studies have focused on the zebrafish embryo due to some advantageous characteristics: its entire developmental phase takes only about four days and takes place outside the mother, allowing a direct visualization of the morphological changes occurring. In such species it has been demonstrated that CNTs do not interfere with proper embryonic development, and the observed 


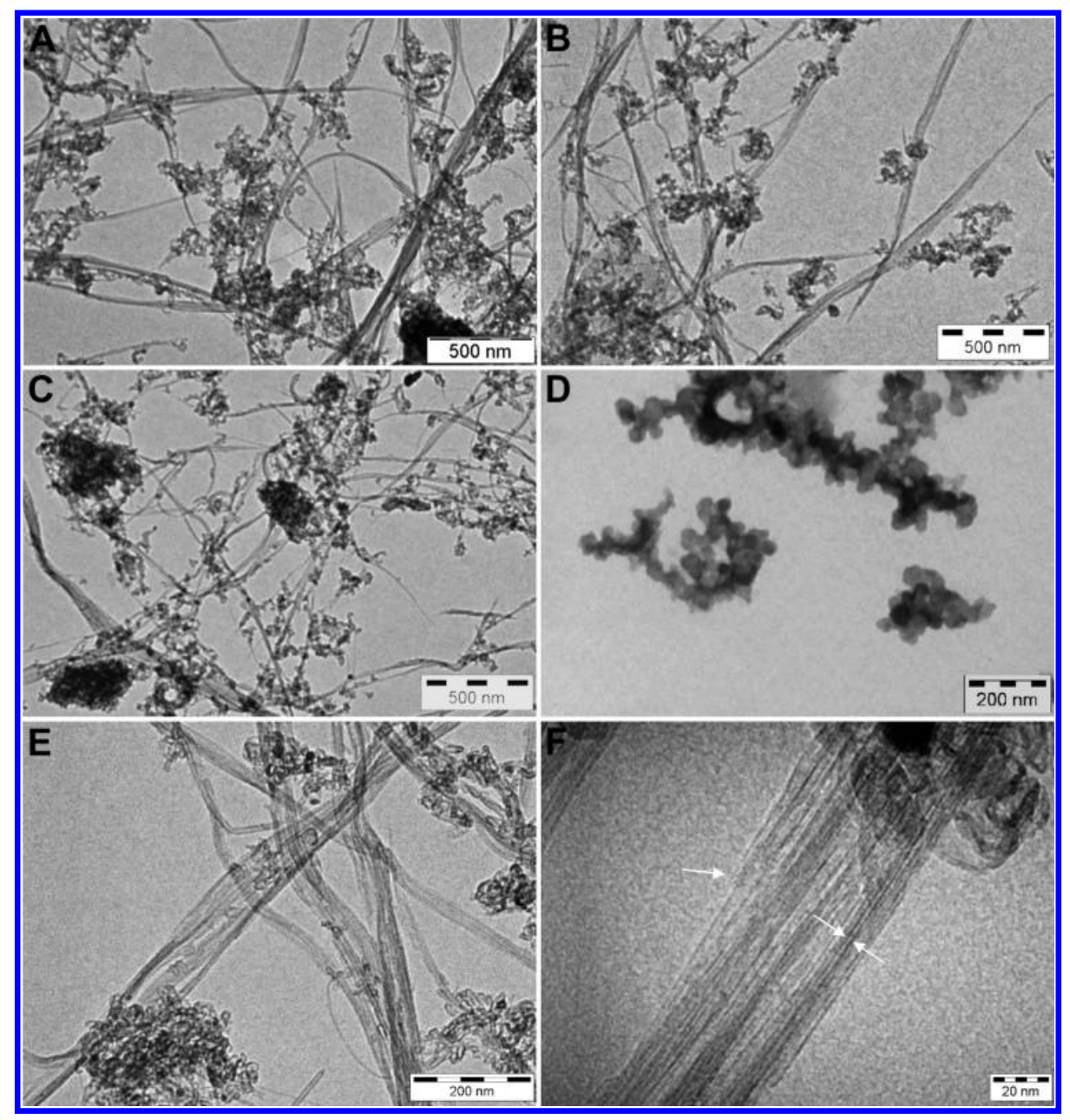

Figure 1. TEM images of (A) p-SWCNTs; (B) o-SWNTs; (C) uo-SWCNTs; and (D) n-CB. SWCNTs appear organized in bundles of different length. A higher abundance of short bundles is observed in uo-SWCNTs. Representative high-magnification images are reported for o-SWNT (E and F). In panel F individual SWCNTs are visible within the bundles (arrows).

hatching delay might be ascribed to $\mathrm{Ni}$ and Co contaminants, rather than the CNTs themselves. ${ }^{17}$ Despite the advantages of the zebrafish model, fish development and embryo morphology differ from that of mammals, and findings for one class cannot be automatically translated to the other. To our knowledge, no studies have been so far performed on the effects of CNTs on mammalian embryonic development, despite the high potential clinical and social relevance of this end point.

The aim of the present study was to assess if the administration of pristine and functionalized singlewall CNTs (SWCNTs) to pregnant mice might adversely affect the development of the postimplantation mouse embryo. In parallel, we have evaluated whether an in vitro test, the embryonic stem cell test ${ }^{20,21}$ might provide reliable predictive information on SWCNTs' embryotoxicity. The EST is an in vitro test validated by the European Centre for the Validation of Alternative Methods (ECVAM) that allows reliably classifying chemical compounds as strong, weak, or nonembryotoxic, without using animal experimentation. The EST has never been applied to evaluate CNTs' embriotoxicity, and there are only limited data on partial application of the test for other nanomaterials. ${ }^{22,23}$ Most importantly, for the evaluation of ENM embryotoxicity, no studies have been reported in which findings from the EST have been matched with in vivo data.

\section{RESULTS AND DISCUSSION}

Three types of SWCNTs have been used: pristine ( $\mathrm{p}$-SWCNTs), oxidized (o-SWCNTs), and ultraoxidized SWCNTs (uo-SWCNTs). p- and o-SWCNTs were purchased, while uo-SWCNTs were prepared in our laboratory by oxidation of o-SWCNT, according to a procedure previously described. ${ }^{24}$ Commercially available nanosized carbon black ( $\mathrm{n}-\mathrm{CB}$ ) was used as a nonfibrous, carbon-based control material. Representative transmission electron microscopy (TEM) images of the samples are shown in Figure 1, and a full characterization of SWCNTs is provided in Table 1. 
For further chemical characterization see the Supporting Information (SI). Pristine and oxidized SWCNTs appeared organized in bundles; aggregates of amorphous carbon were also present. Diameter and length were evaluated by high-resolution TEM analysis and are shown in Table 1. Elemental analysis revealed the presence of $\mathrm{Si}, \mathrm{S}, \mathrm{Fe}, \mathrm{Ca}, \mathrm{Cr}$, and $\mathrm{Co}$ as main impurities in the pristine samples. In the oxidized samples, metals were present only in traces as a consequence of the oxidative treatments used for their preparation (Table1). Since metals leached in the cell culture medium might have contributed to the toxic response, the concentration of metals present in the supernatant of medium in which SWCNTs had been kept in suspension for 10 days (the total duration of the in vitro experiments) was measured by AE-ICP analysis. Among the possible contaminants, only cobalt was found to be released in the medium at final concentrations of 34,5 , and $4 \mu \mathrm{M}$ for p-SWCNTs, o-SWCNTs, and uo-SWCNTs, respectively.

In Vivo Exposure of SWCNTs. Each tested nanomaterial was intravenously (iv) injected in the retrobulbar venous plexus of pregnant females at postcoital day 5.5 (5.5 dpc). At $15.5 \mathrm{dpc}$, animals were sacrificed, and fetuses and placentas were analyzed. The iv administration was preferred to pharyngeal aspiration,

TABLE 1. Physico-chemical Properties of SWCNTs

\begin{tabular}{|c|c|c|c|}
\hline & p-SWCNTs & 0-SWCNTs & uo-SWCNTs \\
\hline elemental analysis ${ }^{a}$ & Si $0.24 \pm 0.06$ & Si $0.28 \pm 0.08$ & Si $0.12 \pm 0.03$ \\
\hline \multirow[t]{5}{*}{$(\% \mathrm{w} / \mathrm{w})$} & S $0.45 \pm 0.04$ & $S 0.48 \pm 0.02$ & S $0.44 \pm 0.10$ \\
\hline & Ca $1.22 \pm 0.31$ & Ca $0.20 \pm 0.004$ & Ca $0.11 \pm 0.02$ \\
\hline & Cr $1.12 \pm 0.26$ & $\operatorname{Cr} 0.63 \pm 0.03$ & Cr $0.27 \pm 0.07$ \\
\hline & Fe $1.10 \pm 0.25$ & Fe $0.26 \pm 0.02$ & Fe $0.14 \pm 0.04$ \\
\hline & Co $3.72 \pm 0.67$ & Co $0.50 \pm 0.02$ & Co $0.22 \pm 0.07$ \\
\hline diameter $(\mathrm{nm})^{b}$ & $2.37 \pm 0.40$ & $1.58 \pm 0.20$ & $1.80 \pm 0.40$ \\
\hline length $(\mu \mathrm{m})^{b}$ & $0.85 \pm 0.42$ & $0.76 \pm 0.70$ & $0.37 \pm 0.17$ \\
\hline weight loss \% (TGA) ${ }^{c}$ & $0.50 \pm 0.50$ & $1.90 \pm 0.50$ & $10.00 \pm 1.00$ \\
\hline acidic sites $(\mathrm{nmol} / \mathrm{mg})^{d}$ & $24.20 \pm 1.10$ & $42.90 \pm 6.70$ & $56.50 \pm 8.40$ \\
\hline extent of defect $\left(I_{D} / I_{G}\right)^{e}$ & 0.90 & 0.10 & 0.10 \\
\hline
\end{tabular}

${ }^{a}$ Evaluated by XRF spectroscopy (mean $\pm \mathrm{SE}$ ). ${ }^{b}$ Evaluated by TEM analysis (mean $\pm S D$ ). ${ }^{C}$ Mean \pm SD. ${ }^{d}$ Acidic sites evaluated as described (ref 4 in SI) (mean \pm SD).

${ }^{e}$ Evaluated by Raman spectroscopy as it does not require general anesthesia, which can by itself affect pregnancy.

Injected mice were daily observed for possible acute or delayed side effects related to the injection of nanomaterials. No deaths, clinically relevant disorders, or evident behavioral changes were recorded.

In the first set of experiments, pregnant females were treated with a relatively high dose of each material (30 $\mu \mathrm{g} / \mathrm{mouse}$ ). At such a dose, all types of SWCNTs, but not n-CB, were able to induce gross fetal morphological abnormalities (Table 2). In addition, a substantial percentage of SWCNT-exposed mice (ranging from $19 \%$ to $31 \%$ ) presented swollen uteri (at least twice the diameter of a nonpregnant normal uterus) with no developed embryos (Table 2), a finding never observed in control females and which we interpreted as evidence of early miscarriages. With the aim to identify the concentration of SWCNTs showing no teratogenic or abortive effects, decreasing doses of the materials were used in subsequent experiments. Interestingly, at $3 \mu \mathrm{g} /$ mouse the percentage of females with early miscarriages greatly decreased, but percentages of mothers with malformed fetuses increased (Table 2). At further lower dosages (0.3 and $0.1 \mu \mathrm{g} / \mathrm{mouse})$, no miscarriages were observed, but mothers carrying fetuses with gross malformations were still present, although in much lower percentages (Table 2). Finally, at the concentration of $0.01 \mu \mathrm{g} /$ mouse no miscarriages or fetal malformations were observed (Table 2). At all dosages, $n-C B$ or vehicle did not exert any teratogenic or abortive effect. $A$ wide variety of malformations was found (Figure $2 A-H$ ), but no differences in their type and severity were observed among the three groups of CNTs and among different concentrations within each group. In some cases the fetuses appeared morphologically normal, but significantly growth retarded (Figure 2E); more often, fetuses with abdominal wall or head deformities or limb hypoplasia (Figure 2B-D and F) were observed. In more abnormal fetuses, a severe retardation in the development of several organs and tissues was associated with an abnormal torsion of the trunk (Figure 2G), or the body plan was profoundly affected

TABLE 2. Summary of the Percentage of Females Whose Pregnancy Was Affected by the Exposure to Different Concentrations of the Three Samples of SWCNTs ${ }^{a}$

\begin{tabular}{|c|c|c|c|c|c|c|c|c|c|c|c|c|c|c|c|c|c|c|c|}
\hline & \multicolumn{4}{|c|}{$30 \mu \mathrm{g} /$ mouse } & \multicolumn{4}{|c|}{$3 \mu \mathrm{g} /$ mouse } & \multicolumn{4}{|c|}{$0.3 \mu \mathrm{g} /$ mouse } & \multicolumn{4}{|c|}{$0.1 \mu \mathrm{g} /$ mouse } & \multicolumn{3}{|c|}{$0.01 \mu \mathrm{g} /$ mouse } \\
\hline & q & $A$ & B & $A+B$ & $q$ & A & B & $A+B$ & ㅇ & A & B & $\mathbf{A}+\mathbf{B}$ & q & A & B & $A+B$ & $q$ & A & B \\
\hline CTRL & 20 & 0 & 0 & 0 & 21 & 0 & 0 & 0 & 21 & 0 & 0 & 0 & 21 & 0 & 0 & 0 & 20 & 0 & 0 \\
\hline$n-C B$ & 23 & 0 & 2 & 0 & 18 & 0 & 0 & 0 & 18 & 0 & 0 & 0 & 20 & 0 & 0 & 0 & 20 & 0 & 0 \\
\hline p-SWCNTs & 21 & 19 & 4.7 & 23.7 & 22 & 4.5 & 27.3 & 31.8 & 19 & 0 & 5.3 & 5.3 & 20 & 0 & 5 & 5 & 18 & 0 & 0 \\
\hline 0-SWCNTs & 20 & 20 & 20 & 40 & 21 & 4.8 & 9.5 & 14.3 & 20 & 0 & 5 & 5 & 18 & 0 & 5.5 & 5.5 & 18 & 0 & 0 \\
\hline uo-SWCNTs & 16 & 31 & 25 & 56 & 20 & 0 & 45 & 45 & 16 & 0 & 18.7 & 18.7 & 23 & 0 & 8.7 & 8.7 & 20 & 0 & 0 \\
\hline
\end{tabular}

${ }^{a} Q$ : number of females analyzed in each group. A: percentage of $q$ with swollen uteri and no fetuses. B: percentage of $q$ with at least one malformed fetus. 


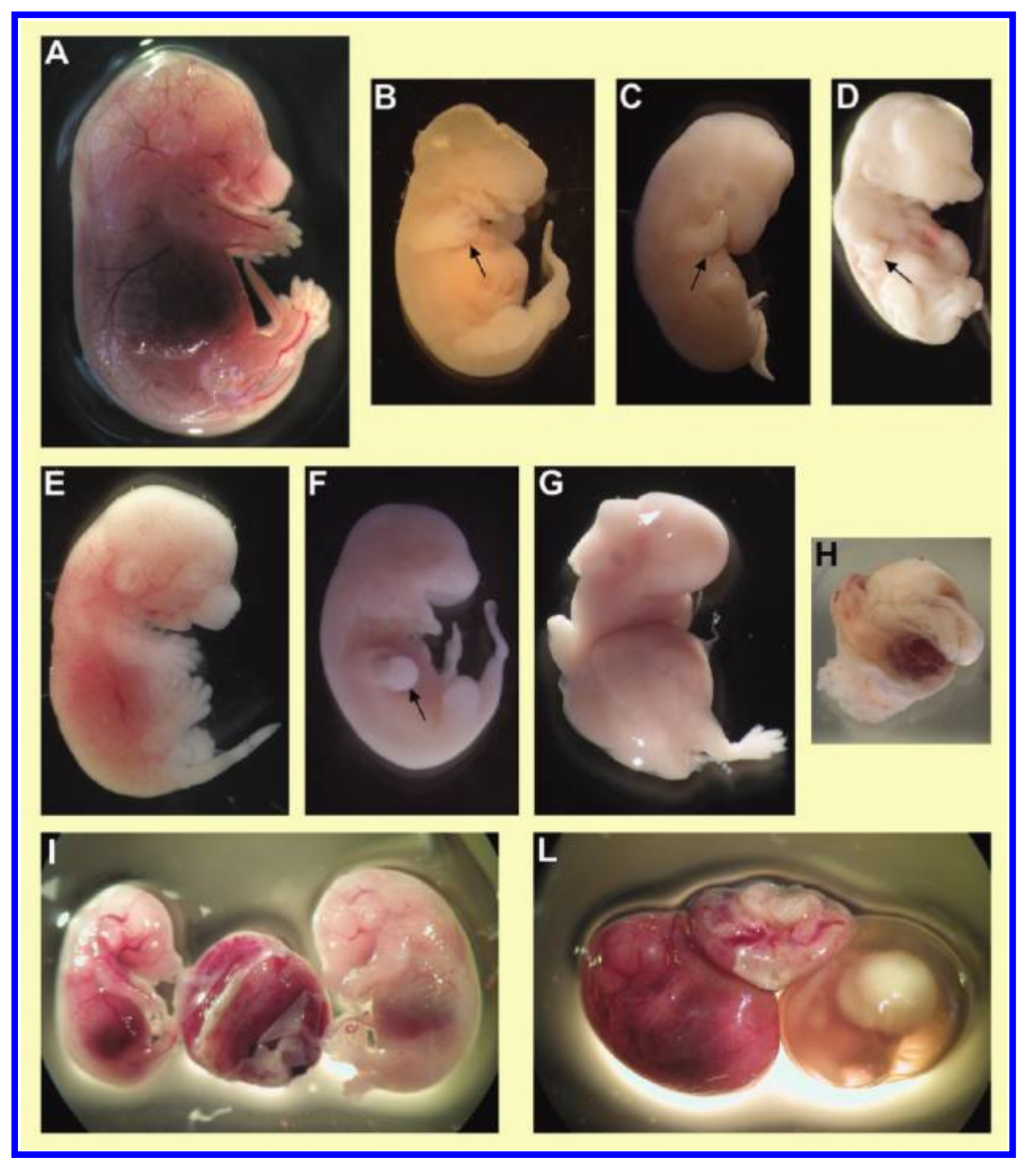

Figure 2. Representative images of $15.5 \mathrm{dpc}$ malformed fetuses from SWCNT-treated mothers. Images refer to $0.3 \mu \mathrm{g} / \mathrm{mouse}$ exposed fetuses, but similar phenotypes were observed at all other effective doses tested. (A) Normal fetus. (B-D) Malformed fetuses recovered from (B) p-, (C) o-, and (D) uo-SWCNT-treated mothers. SWCNT-exposed fetuses appeared developmentally hindered compared to the control. Arrows indicate limbs that appear severely retarded in their development. (E-H) Independently from the type and dosage of SWCNT injected, fetuses with malformations of different severity were recovered. (E) Some fetuses, although smaller, appeared only slightly affected by the treatment, with an overall body plan comparable to that of normal fetuses. (F) Fetuses with a more severe retardation in the development of the limbs and snout were observed. (G) In some cases fetuses missed the crown-rump organization and appeared folded, with swollen abdomen. (H) Small abnormal placentas with a very early developmentally arrested embryo were also recovered. (I, L) Two cases of monozygotic, diamniotic twin fetuses. (I) Twins appear morphologically normal but the fetus on the left side is significantly smaller. (L) In this couple the twin on the right side appears severely developmentally retarded. All photographs are at the same magnification.

and the outlines of the fetus could be barely identified (Figure $2 \mathrm{H}$ )

In a small set of experiments, females were treated with low doses of SWCNTs ( 0.3 and $0.1 \mu \mathrm{g} /$ mouse) at the peri-implantation stage (between day 4.5 and 5 of gestation). Interestingly, in p- and uo-SWCNT-treated females, besides the presence of malformations, a few fetuses were found to share the same placenta (Figure $2 \mathrm{l}$ and $\mathrm{L}$ ). In $80 \%$ of the twinning events, we observed intertwin discordance, encompassing fetal size, feto-placental hemodynamics, and structural defects. Such features resemble vascular pathologies that can complicate monochorial twin pregnancies in humans, such as the "twin to twin transfusion syndrome" and the "twin reversed arterial perfusion sequence" (Figure $2 \mathrm{l}$ and $\mathrm{L}$ ). In both complications one of the twins is developmentally retarded due to blood transfusion via placental vascular anastomosis between the two circulations. ${ }^{25,26}$ Analysis of the gonads revealed that fetuses attached to the same placenta were always of the same sex, a further indication of monozygotic twinning. Monozygotic twinning is an extremely rare event in mice, and in mammals it is widely accepted that its occurrence is a consequence of teratogenic stimuli, ${ }^{27}$ thus indicating that the observed twins might be a result of the teratogenic effect of SWCNTs. No monozygotic twinning was ever observed in n-CBand vehicle-treated females.

At macroscopic analysis, all placentas from malformed fetuses appeared abnormal, whereas no alteration was detected in placentas from normal fetuses. In detail, abnormal placentas presented obvious alterations in size and vascular organization of the labyrinth layer (Figure $3 \mathrm{~A}-\mathrm{C}$ ), characterized by disruption of the 


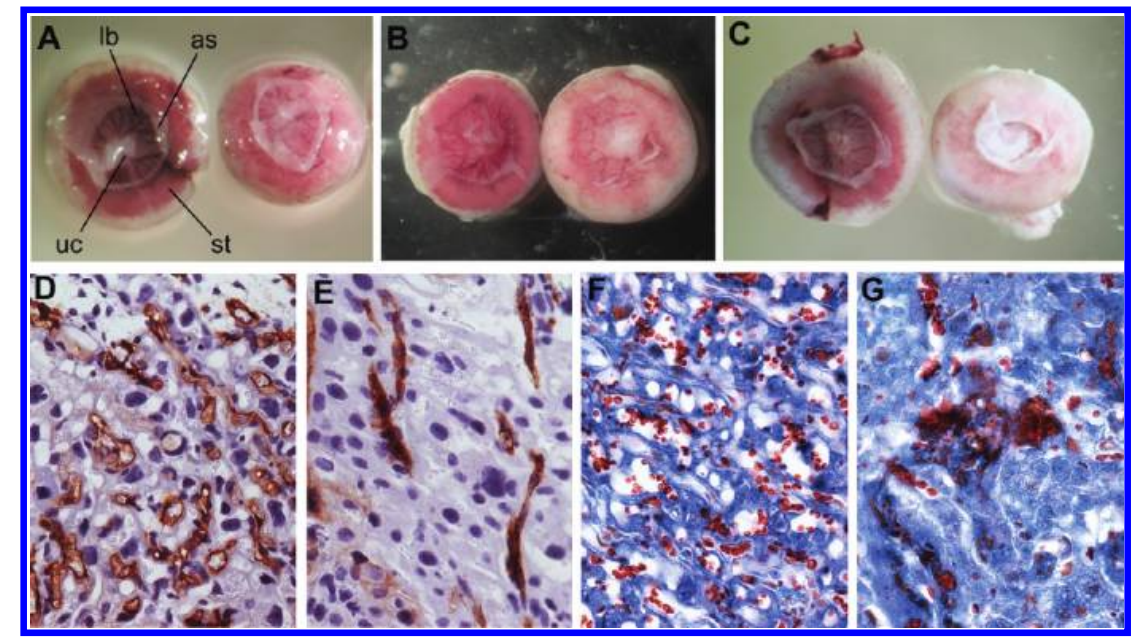

Figure 3. Macroscopic and microscopic analysis of placentas from normal and malformed fetuses. (A-C) Images of placentas from normal (left) and malformed (right) fetuses from (A) p-, (B) o-, and (C) uo-SWCNT-treated mothers. The labyrinth area appears disorganized in the placentas from malformed fetuses. (D, E) Immuno-histochemical analysis using anti-CD31 antibodies shows decreased vascularization in malformed placentas (E) compared to normal ones (D). ( $F$, G) Azan-Mallory staining reveals the presence of fibrin deposition in abnormal placentas (G) that are not detected in normal samples (F). uc: umbilical cord attachment site; lb: labyrinth layer; as: amniotic sac residues; st: maternal spongiotrophoblast.

radial distribution and arborization of the placental vascular bed. Immuno-histochemical analysis using antibodies against the pan-endothelial marker CD31 (Figure 3D and E) and Azan-Mallory staining (Figure 3F and $\mathrm{G}$ ) showed strongly reduced vessel density and branching, as well as areas of fibrin deposition, reflecting the presence of thrombotic vessels. Interestingly, this evident vascular damage, which has never been previously reported in other organs at such very low doses, may be a consequence of the massive angiogenesis occurring in the developing placenta. To verify the hypothesis that the placenta might be particularly vulnerable to exposure to SWCNTs, we performed histological and immuno-histochemical analysis of maternal tissues (liver, lung, kidney, and spleen). No evident tissue alterations were detected (Figure S6 of the $\mathrm{SI}$ ), suggesting that in our experimental conditions the placenta and fetus are mainly affected by the systemic injection of SWCNTs.

The clear association between SWCNT exposure and placental-fetal abnormalities prompted us to evaluate whether SWCNTs could be detected in such tissues, to support the evidence of a direct toxic effect of the nanomaterials. To this end, placentas and fetuses from mothers exposed to the highest dose of SWCNTs ( $30 \mu \mathrm{g} /$ mouse) were analyzed by histological and micro-Raman analyses (Figure S5 of the SI). Neither technique was able to confirm the presence of SWCNTs in the samples. It should be considered, however, that we did not detect SWCNTs in any of the maternal tissues, including lung, liver, and kidney, in which the highest accumulation would be expected (Figure $\mathrm{S} 5$ in the SI). ${ }^{28,29}$ Identification of SWCNTs by Raman and histological analyses has been previously reported, in experiments in which much higher amounts of material had been administered to the animals. ${ }^{29}$ To find out the resolution limit of our detection system, we performed experiments in which increasing amounts of SWCNTs were added to tissue homogenates before Raman spectroscopy analysis. The calculated detection limit was $15 \mu \mathrm{g} / \mathrm{mL}$, corresponding to $0.15 \mu \mathrm{g} / \mathrm{mg}$ of tissue; therefore with the highest dosage injected (30 $\mu \mathrm{g} /$ mouse), we can reach at the maximum a theoretical concentration of $0.001 \mu \mathrm{g} / \mathrm{mg}$, which is well below the detection limit of the technique.

A recognized mechanism through which SWCNTs exert their toxicity is represented by oxidative stress. ${ }^{30}$ Several in vivo and in vitro studies have demonstrated that SWCNTs are able to induce oxidative stress in endothelial arterial cells, leading to inflammatory cell infiltration and platelet-thrombus formation. ${ }^{31,32}$ In addition, it is widely recognized that placental and embryonic tissues are particularly sensitive to oxidative stress, as a consequence of the high cell proliferation rate and concomitant exposure of DNA. ${ }^{33}$ For such reasons it has been proposed that the physiological hypoxia characterizing the first trimester of human gestation might be a way to protect the fetus from teratogenic effects of reactive oxygen species (ROS). ${ }^{34}$ The presence of extensive vascular alterations and of thrombi in our model system (Figure 3D-G) led us to hypothesize that placental damage might be a consequence of oxidative stress. ROS generation was therefore assessed in tissue homogenates from uoSWCNT-treated mothers using dichlorofluorescein-diacetate (DCFDA), a nonfluorescent dye that is oxidized by a variety of ROS to form the highly fluorescent dichlorofluorescein (DCF). ROS were significantly higher in homogenates of placentas from malformed fetuses as compared to samples from vehicle- or 


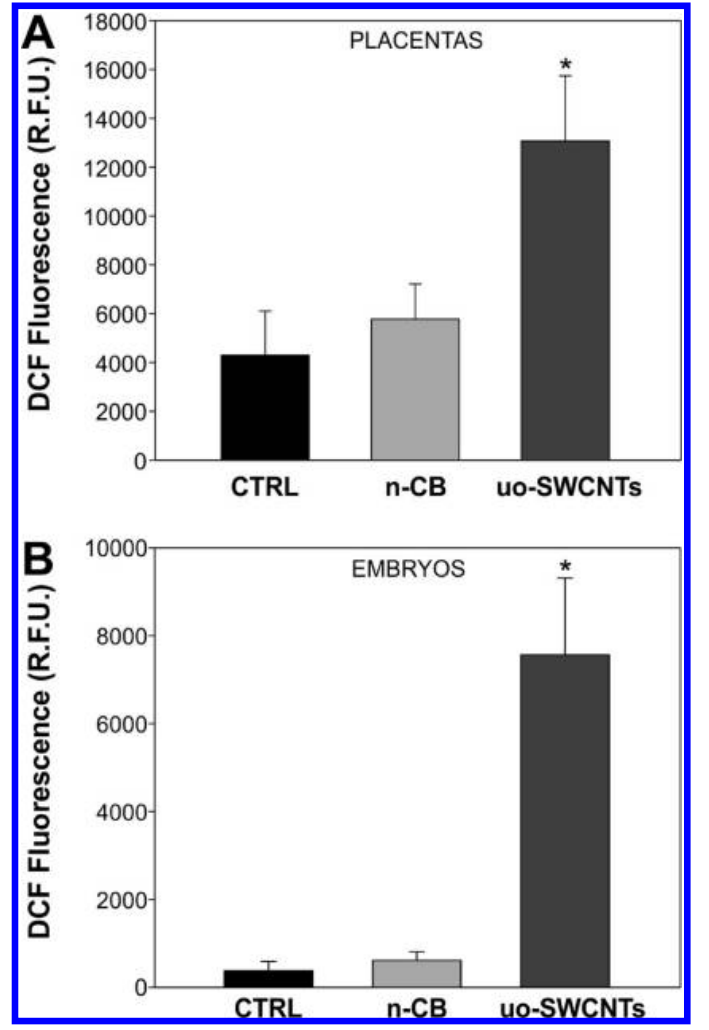

Figure 4. Evaluation of oxidative stress in (A) placentas and (B) fetuses. Values are expressed as relative fluorescence units (RFU) normalized per milligram of protein. Statistical analysis was performed by the ANOVA test $(p<0.01)$.

n-CB-exposed mothers (Figure 4A). Interestingly, ROS levels were also significantly higher in malformed fetuses (Figure 4B).

The occurrence of oxidative stress was evaluated in maternal tissues as well (liver, lung, kidney, and spleen), but no evident differences were observed between exposed and control mice (data not shown).

Embryonic Stem Cell Test. The use of animal testing for routine screening of toxicity of different compounds is time and money consuming and may raise ethical issues. The development of reliable in vitro models would represent a valuable alternative for screening nanomaterial embryotoxicity. The EST is a validated test for predicting embryotoxicity of chemical compounds, ${ }^{20,21}$ and its extension to nanomaterials would be of great importance for evaluating their embryotoxicity without making use of animal studies. To this end, in parallel to the in vivo studies, reliability of the EST for predicting SWCNT embryotoxicity was investigated. In the EST two stable cell lines are used: NIH3T3 fibroblasts (representing differentiated tissue) and mouse embryonic stem (mES) cells (representing embryonic tissue). Three end points are obtained from dose-response curves after 10 days of culture: (i) $50 \%$ inhibition of differentiation of mES cells into contracting myocardial cells $\left(\mathrm{ID}_{50}\right)$, as assessed by

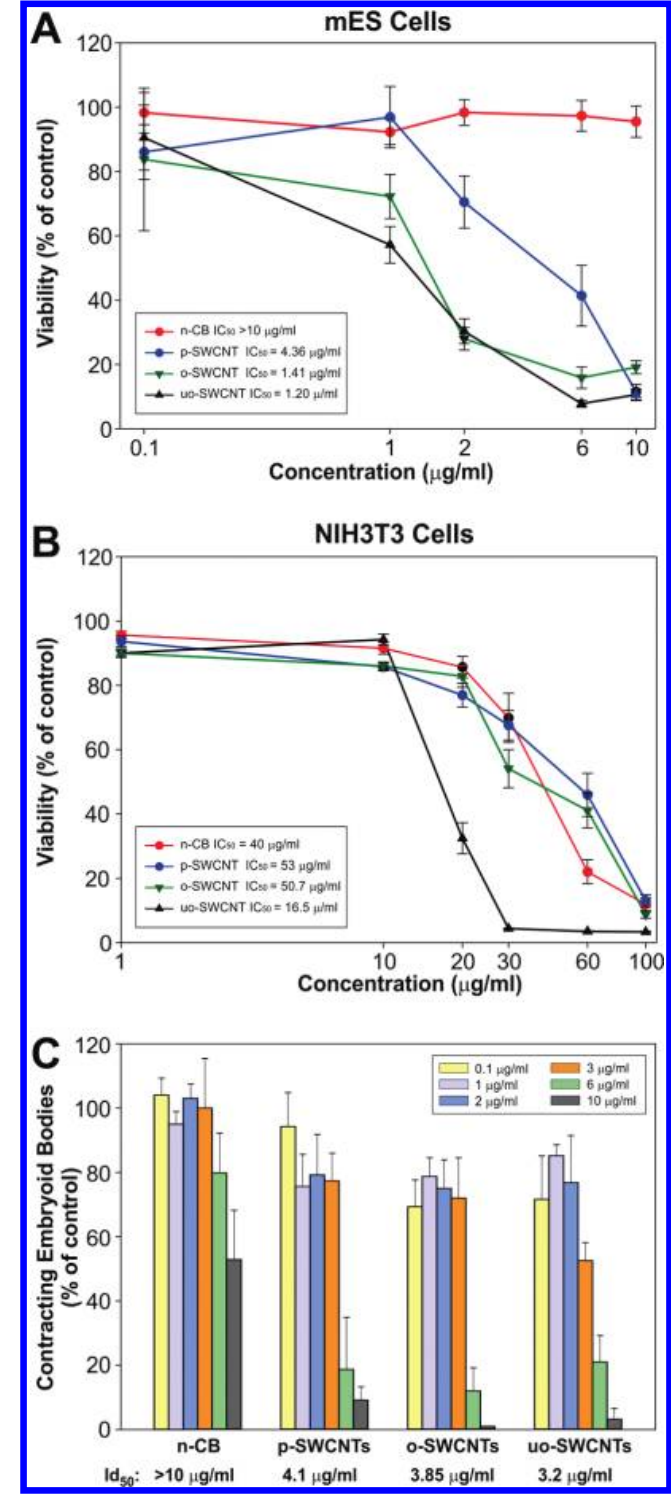

Figure 5. Graphs summarizing the EST results. Viability of $\mathrm{mES}(\mathrm{A})$ and NIH3T3 (B) cells in the presence of increasing concentrations of $n-C B$ and SWCNTs was measured using the WST- 1 assay. The calculated $I_{50}$ values are reported in boxes at the lower left corner. The ability of SWCNTs to impair mES differentiation into contracting EBs was evalu ated by direct visualization of beating areas under a light microscope. (C) The calculated $\mathrm{ID}_{50}$ for each material is reported at the bottom of the graph. Values are means \pm standard error of at least 5 independent experiments.

morphological analysis of beating cell areas (contracting embryoid bodies, EBs); (ii) 50\% inhibition of proliferation of mES (IC 5OES $_{\text {) }}$ and (iii) of NIH3T3 cells $\left(\mathrm{IC}_{\text {503т3 }}\right)$. An algorithm eventually integrates these three values to obtain an evaluation of predicted embryotoxicity. As required by the EST standardized protocol, ${ }^{20,21}$ due to the higher sensitivity of mES to toxic agents, as compared to NIH3T3 differentiated cells, different concentrations of the tested compound are to be used for the two cell lines in order to obtain 
the IC $C_{50}$ values. In our experiment, the effect of SWCNTs on cell proliferation was studied by growing the cells in medium containing the same carbon compounds used in the in vivo experiments, at concentrations between 0.1 and $10 \mu \mathrm{g} / \mathrm{mL}$ for $\mathrm{mES}$ cells and 1 and $100 \mu \mathrm{g} / \mathrm{mL}$ for $\mathrm{NIH} 3 \mathrm{~T} 3$ cells. After 10 days of culture, cell viability was measured by a colorimetric assay (WST-1) and the IC $\mathrm{C}_{50}$ values were calculated for each carbon compound in each cell line (Figure 5A and B). The ID 50 was obtained by culturing $\mathrm{mES}$ cells under conditions stimulating differentiation into EBs in the presence of nanoparticle concentrations ranging from 0.1 to $10 \mu \mathrm{g} / \mathrm{mL}$. For each material, no significant decrease of $\mathrm{mES}$ cell viability was observed at $0.1 \mu \mathrm{g} / \mathrm{mL}$; o- and uo-SWCNTs decreased viability to about $70 \%$ and $60 \%$, respectively, at $1 \mu \mathrm{g} / \mathrm{mL}$, to less than $40 \%$ at $2 \mu \mathrm{g} / \mathrm{mL}$, and down to approximately zero at higher concentrations. The cal-

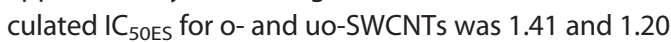
$\mu \mathrm{g} / \mathrm{mL}$, respectively. The effect of p-SWCNT exposure was first detected at $2 \mu \mathrm{g} / \mathrm{mL}$, and only at the highest dose $(10 \mu \mathrm{g} / \mathrm{mL})$ was it comparable to what was observed for the oxidized materials. For such material the IC $C_{50 E S}$ was $4.36 \mu \mathrm{g} / \mathrm{mL}$. $\mathrm{n}$-CB showed no significant effect on $\mathrm{mES}$ at any of the tested doses.

A different pattern was observed with NIH3T3 cells; for each tested material no effect was detected up to $10 \mu \mathrm{g} / \mathrm{mL}$, a dose killing almost $100 \%$ of $\mathrm{mES}$. For such reasons, in order to obtain the $\mathrm{IC}_{503 т 3}$ value, higher concentrations were tested. At $20 \mu \mathrm{g} / \mathrm{mL}$ uo-SWCNT exposure caused a sharp decrease in viability (down to $30 \%$ ), and almost no cell survival was recorded at 30 $\mu \mathrm{g} / \mathrm{mL}$. The other three types of materials showed a similar pattern, with a progressive decrease in viability starting at $20 \mu \mathrm{g} / \mathrm{mL}$ and reaching the same level as uoSWCNTs at the highest dose.

Regarding the effect of the four tested materials on $m E S$ differentiation into contracting EBs, $n-C B$ was ineffective up to $3 \mu \mathrm{g} / \mathrm{mL}$, and the percentage of contracting EBs was decreased to $55 \%$ at the highest dose (ID ${ }_{50}$ not measurable). By contrast, the three types of SWCNTs showed a marked inhibitory effect starting at $6 \mu \mathrm{g} / \mathrm{mL}$, with contracting EBs being reduced to about $20 \%$. At the highest dose $(10 \mu \mathrm{g} / \mathrm{mL})$ almost no contracting $\mathrm{EBs}$ were detected. The calculated $\mathrm{ID}_{50}$ values for $\mathrm{p}$-, $\mathrm{O}-$, and uo-SWCNTs were 4.1, 3.85, and $3.2 \mu \mathrm{g} / \mathrm{mL}$, respectively.

The EST algorithm classified all three types of SWCNTs as "strong embryotoxic". By contrast, the $\mathrm{IC}_{50}$ ES and $\mathrm{ID}_{50}$ for $\mathrm{n}-\mathrm{CB}$ were above the tested range, so that no classification was possible for such nanomaterial. These findings are in accordance with the in vivo results, thus suggesting that the EST, currently used for predicting embryotoxicity of soluble chemical compounds, may have the same accuracy for nanomaterials. Very few and partial attempts to apply the EST to other nanoparticles have been reported, ${ }^{22,23}$ and none of such studies have matched in vitro data to in vivo data. To date, this is the first time that the full EST protocol has been applied to the study of nanomaterials.

The possible leakage to the medium of contaminants from SWCNTs might have affected our results. To verify this hypothesis, we performed a set of experiments in which ES and NIH3T3 cells were cultured in SWCNT-conditioned medium (see SI). No effect on viability of the two cell lines or development of contracting EBs was observed, as compared to control medium (Figure S7). These findings suggest a negligible effect on the in vitro results of contaminants released from SWCNTs.

\section{CONCLUSION}

Our in vitro and in vivo results suggest that SWCNTs may act as embryotoxic agents in mammals. The concordance of in vitro and in vivo data is generally considered as an important issue when assessing toxicological properties of any material, since validation of the in vitro findings may allow further in vivo studies to be drastically reduced or omitted.

In the in vivo experiments, teratogenicity was still observed at a dosage as low as of $100 \mathrm{ng} /$ mouse. On the basis of data from the literature on the biodistribution of SWCNTs, ${ }^{29,35}$ it is reasonable to assume that only a minor fraction of the injected material may reach the placenta and the fetus. The lowest dose found to be effective in the present study is several orders of magnitude lower than what has been previously reported in other toxicological experiments, with different end points. As an example, pro-carcinogenic properties of CNTs have been suggested by a study in which a 500 times higher amount was directly injected into the target tissue. ${ }^{10}$ Our findings therefore suggest that the fetus is highly susceptible to the exposure to these materials. We could not discriminate between a direct or indirect effect of SWCNTs to the placenta and/or the fetus, since the nanotubes were not detectable in such tissues, probably due to the low administered dose. However, an oxidative damage, typically linked to SWCNT pathogenic mechanisms, ${ }^{30}$ was observed in both malformed placentas and fetuses. Further studies might be performed, evaluating whether and at which dosage the in vivo direct injection in the placenta of SWCNTs may reproduce the findings observed in our experiment.

Although no differences in the type and severity of malformations were detected in fetuses exposed to the three different classes of SWCNTs, a higher frequency of fetal malformations was observed in uo-SWCNTtreated mothers. Since the functionalization process strongly reduces the presence of contaminants, which are considered as one of the main players in nanoparticle toxicity, the most marked embryotoxic effect of uo-SWCNTs seems to be determined by some intrinsic physicochemical characteristics of the material itself. $A$ 
higher toxicity of oxidized, as compared to pristine, CNTs has been previously reported for both in vitro and in vivo models, $8,36,37$ and it is usually ascribed to a better dispersion of the material in the medium and its consequent higher bioavailability. ${ }^{8,36,37}$ Although we observed a similar size distribution of aggregates between pristine and oxidized SWCNTs, we cannot exclude that monodispersed SWCNTs were more abundant in oxidized SWCNTs. Apart from dispersion, the higher negative charge and hydrophilicity of oxidized CNTs, which is a consequence of the introduction of oxygenated functionalities, may also play a role. ${ }^{37}$

The pathophysiologic mechanism by which SWCNTs adversely affect pregnancy has been only partially explored in the present study, but the strict association between embryonic developmental retardation and placental damage suggests a role of the placenta in mediating the embryotoxic effect. Our in vitro studies, however, show that embryonic tissues are strongly sensitive to SWCNT damage, and therefore a direct effect on the embryo in vivo may be hypothesized. Evidence that nanoparticles can cross the blood-placental barrier has been reported. ${ }^{38,39}$ These data, however, refer to polystyrene beads and gold nanoparticles and cannot be directly applied to SWCNTs, given the different shape and length of these nanomaterials. Data on the ability of SWCNTs to cross the placental barrier are needed.

In conclusion, our results suggest that exposure to SWCNTs may represent a potential risk for pregnant women, especially in the occupational setting, where the risk of accidental exposure may become real in light of the foreseen increased production of ENM in the near future.

Further studies are needed in order to fully understand the underlying mechanism by which CNTs (especially oxidized CNTs) can induce embryotoxicity and to confirm the reliability of the EST in predicting embryotoxicity for other ENMs.

\section{MATERIALS AND METHODS}

Materials and Reagents. p-SWCNT and o-SWCNTs were purchased from Cheap Tubes Inc. (Brattleboro, VT). Carbon black was purchased from Cabot Corporation (Boston, MA). All reagents were of analytical grade and were purchased from Sigma-Aldrich (St. Louis, MO), unless otherwise specified. Deionized water was produced by a Milli-Q system (Millipore, Billerica, MA) and was used for all chemical procedures.

Preparation of uo-SWCNTs. uo-SWCNTs were prepared by acidic treatment of the commercially available o-SWCNTs, In details, o-SWCNTs were treated with a solution of $\mathrm{HNO}_{3} / \mathrm{H}_{2} \mathrm{SO}_{4} 1: 3(\mathrm{v} / \mathrm{v})$ for $8 \mathrm{~h}$ at $50{ }^{\circ} \mathrm{C}$, in an ultrasonic bath (at $50 \mathrm{~W}$ ). After the treatment, the powder was washed with bidistilled water and separated by centrifugation until neutrality was reached. Finally, the powder was dried overnight at $100^{\circ} \mathrm{C}$ and stored at room temperature in a tightly closed vial.

Morphological Characterization. Morphological characterization of the three types of SWCNTs and n-CB was performed by transmission electron microscopy (Philips, CM120, with a LaB6 filament, $100 \mathrm{kV}$ ). For the analysis, nanomaterial powders were first dispersed in ethanol ( $1 \mathrm{mg} / 5 \mathrm{~mL}$ ) using an ultrasonic bath for $1 \mathrm{~h}$ at room temperature. A perforated carbon Formvar film supported by copper grids $(\varphi=3 \mathrm{~mm}$ ) was dipped in the suspensions for a few seconds and the solvent evaporated under an infrared lamp. The substrate was then assembled on aluminum stubs with carbon tape.

In order to clearly distinguish between bundles of SWCNTS and single tubes, the length was measured at a magnification of $31000 \times$. With such a method CNTs that cross the borders of the visual field cannot be considered, so that the overall length of the three types of SWCNTs might have been underestimated. Length was calculated as the mean of SWCNTs that were entirely present in the visual field $( \pm S D)$.

In Vivo Experimental Procedures. All animal procedures were approved by the Institutional Animal Care and Use Committee and comply with European rules (116/92). Animals used in the present study were six to eight week old females of the CD1 outbred strain (Charles River, Calco, Italy), considered as a general multipurpose model for in vivo studies. All animals were housed and mated under standard laboratory conditions. Their weight was between 30 and $35 \mathrm{~g}$. Females at day 5.5 of gestation were injected in the retrobulbar plexus with $\mathrm{n}-\mathrm{CB}, \mathrm{p}$ $\mathrm{o}-$, uo-SWCNTs (stock solutions of $1 \mathrm{mg} / \mathrm{mL}$ in DMEM containing $10 \mathrm{mg} / \mathrm{mL}$ BSA), or control medium (DMEM-BSA). Saline solution was used to adjust the final injection volume to $100 \mu \mathrm{L}$
The retrobulbar injection is a valuable alternative to the tail vein injection route and is recommended for small laboratory animals. ${ }^{40}$ Such procedure is much less technically challenging than tail vein injection, and to an experienced operator no anesthesia is required, as hand restraint is sufficient; in addition, there is no need for warming preparation of the animal, and the whole procedure is only a matter of seconds. In our experiments, the mouse was immobilized on absorbent paper, keeping it motionless. Using a $1 \mathrm{cc}$ syringe and a 27 gauge needle, a volume of $100 \mu \mathrm{L}$ was gently injected in the center of the retroorbital sinus of the right eye. No local complications related to the procedure were observed. Ten days later, control and treated females were euthanized by carbon dioxide, and fetuses and their placentas collected and analyzed for the presence of malformations under a stereomicroscope. Fetuses that presented evident morphological abnormalities were photographed and then fixed with their placentas in $4 \%$ paraformaldehyde together with a morphologically normal sibling for subsequent histochemical and immuno-histochemical analysis. In parallel, fetuses and placentas from control mothers were analyzed.

Histochemical and Immuno-histochemical Analysis. Fetuses and placentas were processed for paraffin embedding, following standard procedures. Five-micrometer sections of placentas from normal and malformed fetuses were stained with Azan Mallory in order to visualize the presence of fibrin deposition ${ }^{41}$ Parallel sections were immuno-stained with anti-CD31 antibodies (BD Biosciences, Franklin Lakes, NJ) to outline the vascular network. Further experimental details are reported in the Supporting Information.

Cell Culture. Mouse ES cell line D3 and NIH3T3 cells were purchased from ATCC (America Type Tissue Collection, Manassas, VA). Both cell lines were maintained in culture following standard protocols (for details see $\mathrm{SI}$ ). For proliferation assay experiments, $500 \mathrm{mES}$ or NIH3T3 cells were plated in each well of a 96-well plate in the presence of different concentration of the three types of SWCNTs, of $n-C B$ or BSA. For mES cells the concentrations tested were $0.1,1,2,6$, and $10 \mu \mathrm{g} / \mathrm{mL}$; for NIH3T3 cells 10 times higher concentrations were used. On day 10 , proliferation of the cells was evaluated using the WST-1 colorimetric assay following the manufacturer's specifications (Cell Proliferation Reagent WST-1, Roche Diagnostics, Indianapolis, IN) (for details see SI). Due to the production of water-soluble formazan salts, such test has been proven compatible with the use of SWCNTs. ${ }^{42}$ For mES cell differentiation into embryoid bodies the "hanging drops" protocol was followed according to 
the EST guidelines. On day 10 the presence of spontaneous contraction of the embryoid bodies was visually evaluated under the light microscope. Further experimental details are reported in the Supporting Information.

Evaluation of Oxidative Stress. Production of ROS was measured in tissue homogenates by the conversion of nonfluorescent $2^{\prime}, 7^{\prime}$-dichlorofluorescein-diacetate to the highly fluorescent $2^{\prime}, 7^{\prime}$-dichlorofluorescein. Briefly, the assay measures the oxidative conversion of stable, nonfluorescent DCF-DA to the highly fluorescent DCF in the presence of esterases and ROS. ${ }^{43}$ Tissues were homogenized in homogenization buffer $(20 \mathrm{mM}$ glycerophosphate, $20 \mathrm{mM} \mathrm{NaF}, 2 \mathrm{mM}$ sodium orthovanadate, $1 \mathrm{mM}$ EDTA, $0.5 \mathrm{mM}$ phenylmethylsulfonyl fluoride, $1 \mathrm{mM}$ pepstatin $80 \mathrm{mg} / \mathrm{L}$ trypsin inhibitor, and $100 \mathrm{mM}$ Tris-Cl, $\mathrm{pH}$ 7.4) and centrifuged at $900 \mathrm{~g}$ at $4{ }^{\circ} \mathrm{C}$ for $15 \mathrm{~min}$. The supernatants were further centrifuged at $12000 \mathrm{~g}$ for an additional $15 \mathrm{~min}$ and were assayed for protein content by the BioRad protein assay method (BioRad Laboratories GmbH, Munchen, Germany). A $100 \mu \mathrm{g}$ amount of proteins of each sample was incubated with a final concentration of $25 \mu \mathrm{M}$ DCF-DA in a 96-well plate at $37{ }^{\circ} \mathrm{C}$ Measurements were taken at 0 and $30 \mathrm{~min}$ with excitation and emission wavelengths of 495 and $530 \mathrm{~nm}$, respectively, using a SPECTRAmax GEMINI-XS microplate fluorescence reade (Molecular Devices, Sunnyvale, CA). Values are expressed as relative fluorescence units (RFU) normalized per milligram of protein.

Acknowledgment. We would like to thank Graziano Bonelli and Gabriele Rossi for excellent technical assistance, Graziana Palmieri for help with the retrobulbar injections, and Marilena Carbone for help with part of the TEM studies. We also would like to thank Sergio Ferrazzani and Silvia Salvi for help with the interpretation of the twinning pathologies. This study was supported by The Regione Piemonte (grant number D14-2005 to E.C. and CIPE 2006 project "NANOSAFE"), the Compagnia San Paolo (grant to the NIS Center), and the University of Tor Vergata funding for scientific projects (RSA 2009).

Conflict of Interest: The authors declare no conflict of interest.

Supporting Information Available: Additional results and further information on production and characterization of the nanomaterials. This material is free of charge via the Internet at http://pubs.acs.org.

\section{REFERENCES AND NOTES}

1. Qiu, X.; Freitag, M.; Perebeinos, V.; Avouris, P. Photoconductivity Spectra of Single-Carbon Nanotubes: Implications on the Nature of Their Excited States. Nano Lett. 2005, 5, 749-752.

2. Ferrari, M. Cancer Nanotechnology: Opportunities and Challenges. Nat. Rev. Cancer 2005, 5, 161-171.

3. Ferriera, L.; Karp, J. M.; Nobre, L.; Langer, R. New Opportunities: the Use of Nanotechnologies to Manipulate and Track Stem Cells. Cell Stem Cell 2008, 3, 136-146.

4. Rivera Gil, P.; Oberdörster, G.; Elder, A.; Puntes, V.; Parak, W. J. Correlating Physico-Chemical with Toxicological Properties of Nanoparticles: the Present and the Future. ACS Nano 2010, 4, 5527-5531.

5. Sanchez, V. C.; Pietruska, J. R.; Miselis, N. R.; Hurt, R. H.; Kane, A. B. Biopersistence and Potential Adverse Health Impacts of Fibrous Nanomaterials: What Have We Learned from Asbestos? Wiley Interdiscip Rev. Nanomed. Nanobiotechnol. 2009, 1, 511-529.

6. Kagan, V. E.; Tyurina, Y. Y.; Tyurin, V. A.; Konduru, N. V.; Potapovich, A. I.; Osipov, A. N.; Kisin, E. R.; Schwegler-Berry, D.; Mercer, R.; Castranova, V.; et al. Direct and Indirect Effects of Single Walled Carbon Nanotubes on RAW 264.7 Macrophages: Role of Iron. Toxicol. Lett. 2006, 165, 88-100.

7. Pulskamp, K.; Diabaté, S.; Krug, H. F. Carbon Nanotubes Show No Sign of Acute Toxicity but Induce Intracellular Reactive Oxygen Species in Dependence on Contaminants. Toxicol. Lett. 2007, 168, 58-74.

8. Bottini, M.; Bruckner, S.; Nika, K.; Bottini, N.; Bellucci, S. Magrini, A.; Bergamaschi, A.; Mustelin, T. Multi-Walled
Carbon Nanotubes Induce T Lymphocyte Apoptosis. Toxicol. Lett. 2006, 160, 121-126.

9. Sato, Y.; Yokoyama, A.; Shibata, K.; Akimoto, Y.; Ogino, S.; Nodasaka, Y.; Kohgo, T.; Tamura, K.; Akasaka, T.; Uo, M.; et al. Influence of Length on Cytotoxicity of Multi-Walled Carbon Nanotubes against Human Acute Monocytic Leukemia Cell Line THP-1 In Vitro and Subcutaneous Tissue of Rats In Vivo. Mol. Biosyst. 2005, 1, 176-182.

10. Poland, C. A.; Duffin, R.; Kinloch, I.; Maynard, A.; Wallace, W. A.; Seaton, A.; Stone, V.; Brown, S.; Macnee, W. Donaldson, K. Carbon Nanotubes Introduced into the Abdominal Cavity of Mice Show Asbestos-Like Pathogenicity in a Pilot Study. Nat. Nanotechnol. 2008, 3, 423-428.

11. Fenoglio, I.; Tomatis, M.; Lison, D.; Muller, J.; Fonseca, A.; Nagy, J. B.; Fubini, B. Reactivity of Carbon Nanotubes: Free Radical Generation or Scavenging Activity? Free Radical Biol Med. 2006, 40, 1227-1233.

12. Fenoglio, I.; Greco, G.; Tomatis, M.; Muller, J.; RaymundoPiñero, E.; Béguin, F.; Fonseca, A.; Nagy, J. B.; Lison, D.; Fubini, B. Structural Defects Play a Major Role in the Acute Lung Toxicity of Multiwall Carbon Nanotubes: Physicochemical Aspects. Chem. Res. Toxicol. 2008, 21, 1690-1697.

13. Muller, J.; Huaux, F.; Fonseca, A.; Nagy, J. B.; Moreau, N.; Delos, M.; Raymundo-Piñero, E.; Béguin, F.; Kirsch-Volders, M.; Fenoglio, l.; et al. Structural Defects Play a Major Role in the Acute Lung Toxicity of Multiwall Carbon Nanotubes: Toxicological Aspects. Chem. Res. Toxicol. 2008, 21, 1698-1705.

14. Fubini, B.; Ghiazza, M.; Fenoglio, I. Physico-Chemical Features of Engineered Nanoparticles Relevant to Their Toxicity. Nanotoxicology 2010, 4, 347-363.

15. Shvedova, A. A.; Kisin, E. R.; Porter, D.; Schulte, P.; Kagan, V. E.; Fadeel, B.; Castranova, V. Mechanisms of Pulmonary Toxicity and Medical Applications of Carbon Nanotubes: Two Faces of Janus? Pharmacol Ther. 2009, 121, 192-204.

16. Lacerda, L.; Bianco, A.; Prato, M.; Kostarelos, K. Carbon Nanotubes as Nanomedicines: from Toxicology to Pharmacology. Adv. Drug Delivery Rev. 2006, 58, 1460-1470.

17. Cheng, J.; Flahaut, E.; Cheng, S. H. Effect of Carbon Nanotubes on Developing Zebrafish (Danio rerio) Embryos. Environ. Toxicol. Chem. 2007, 26, 708-716.

18. Cheng, J.; Chan, C. M.; Veca, L. M.; Poon, W. L.; Chan, P. K.; Qu, L.; Sun, Y. P.; Cheng, S. H. Acute and Long-Term Effects after Single Loading of Functionalized Multi-Walled Carbon Nanotubes into Zebrafish (Danio rerio). Toxicol. Appl. Pharmacol. 2009, 235, 216-225.

19. Asharani, P. V. Serina N. G. Nurmawati, M. H. Wu, Y. L. Gong, Z.; Valiyaveettil, S. Impact of Multi-Walled Carbon Nanotubes on Aquatic Species. J. Nanosci. Nanotechnol. 2008, 8, 3603-3609.

20. Scholz, G.; Spielman, H. Embryonic Stem Cell Test (EST). INVITTOX 2000, 113, 1-33.

21. Genschow, E. Spielmann, $\mathrm{H} \cdot$ Scholz, G. Pohl, I-Seiler, A. Clemann, N.; Bremer, S.; Becker, K. Validation of the Embryonic Stem Cell Test in the International ECVAM Validation Study on Three In Vitro Embryotoxicity Tests. Altern. Lab. Anim. 2004, 32, 209-244.

22. Park, M. V.; Annema, W.; Salvati, A.; Lesniak, A.; Elsaesser, A.; Barnes, C.; McKerr, G.; Howard, C. V.; Lynch, I.; Dawson, K. A.; et al. In Vitro Developmental Toxicity Test Detects Inhibition of Stem Cell Differentiation by Silica Nanoparticles. Toxicol. Appl. Pharmacol. 2009, 240, 108-116.

23. Di Guglielmo, C.; López, D. R.; De Lapuente, J.; Mallafre, J. M.; Suàrez, M. B. Embryotoxicity of Cobalt Ferrite and Gold Nanoparticles: a First In Vitro Approach. Reprod. Toxicol. 2010, 30, 271-276.

24. Cataldo, F.; Compagnini, G.; Patanè, G.; Ursini, O.; Angelini, G.; Rebernik Ribic, P.; Margaritondo, G.; Cricenti, A.; Palleschi, G.; Valentini, F. Graphene Nanoribbons Produced by the Oxidative Unzipping of Single-Wall Carbon Nanotubes. Carbon 2010, 48, 2596-2602.

25. Suzuki, K.; Kobayashi, M.; Shiraishi, Y.; Sugiura, Y.; Kobayashi, K: Goto, S. Placental Histology in Twin-Twin Transfusion Syndrome (TTS) in the Mouse. Placenta 1999, 20), 485-491.

26. Van Allen, M. I.; Smith, D. W.; Shepard, T. H. Twin Reversed Arterial Perfusion (TRAP) Sequence: a Study of 14 Twin 
Pregnancies with Acardius. Semin. Perinatol. 1983, 7, 285-293.

27. Kaufman, M. H. The Embryology of Conjoined Twins. Childs Nerv. Syst. 2004, 20, 508-525.

28. Yang, S. T.; Guo, W.; Lin, Y.; Deng, X. Y.; Wang, H. F.; Sun, H. F.; Liu, Y. F.; Wang, X.; Wang, W.; Chen, M.; et al. Biodistribution of Pristine Single-Walled Carbon Nanotubes In Vivo. J. Phys. Chem. C 2007, 111, 17761-1776.

29. Yang, S. T.; Wang, X.; Jia, G.; Gu, Y.; Wang, T.; Nie, H.; Ge, C.; Wang, H.; Liu, Y. Long-Term Accumulation and Low Toxicity of Single-Walled Carbon Nanotubes in Intravenously Exposed Mice. Toxicol. Lett. 2008, 181, 182-189.

30. Donaldson, K.: Aitken, R.: Tran, L.: Stone, V.: Duffin, R Carbon Nanotubes: a Review of Their Properties in Relation to Pulmonary Toxicology and Workplace Safety. Toxicol. Sci. 2006, 92, 5-22.

31. Zhiqing, L.; Zhuge, X.; Fuhuan, C.; Danfeng, Y.; Huashan, Z.; Bencheng, L.; Wei, Z.; Huanliang, L.; Xin, S. ICAM-1 and VCAM-1 Expression in Rat Aortic Endothelial Cells After Single-Walled Carbon Nanotube Exposure. J. Nanosci. Nanotechnol. 2010, 10, 8562-8574.

32. Li, Z.; Hulderman, T.; Salmen, R.; Chapman, R.; Leonard, S. S.; Young, S. H.; Shvedova, A.; Luster, M. I.; Simeonova, P. P. Cardiovascular Effects of Pulmonary Exposure to SingleWall Carbon Nanotubes. Environ. Health Persp. 2007, 115, 377-382.

33. Burton, G. J.; Hempstock, J.; Jauniaux, E. Oxygen, Early Embryonic Metabolism and Free Radical Mediated Embryopathies. Reprod. Biomed. Online 2003, 6, 84-96.

34. Jauniaux, E.; Gulbis, B. Burton, G. J. The Human First Trimester Gestational Sac Limits Rather Than Facilitates Oxygen Transfer to the Foetus--a Review. Placenta 2003, 24), S86-93.

35. Liu, Z.; Davis, C.; Cai, W.; He, L.; Chen, X.; Dai, H. Circulation and Long-Term Fate of Functionalized, Biocompatible, Single-Walled Carbon Nanotubes in Mice Probed by Raman Spectroscopy. Proc. Natl. Acad. Sci. U. S. A. 2008, 105, 14101415.

36. Saxena, R. K.; Williams, W.; McGee, J. K.; Daniels, M. J.; Boykin, E.; Gilmour, M. I. Enhanced In Vitro and In Vivo Toxicity of Poly-Dispersed Acid-Functionalized Single-Wall Carbon Nanotubes. Nanotoxicology 2007, 1, 291-300.

37. Panessa-Warren, B. J.; Maybe, M. M.; Warren, J. B.; Crosson, K. M. Single Walled Carbon Nanotube Reactivity and Cytotoxicity Following Extended Aqueous Exposure. Environ. Pollut. 2009, 157, 1140-1151.

38. Wick, P.; Malek, A.; Manser, P.; Meili, D.; Maeder-Althaus, X.; Diener, L.; Diener, P. A.; Zisch, A.; Krug, H. F.; von Mandach, U. Barrier Capacity of Human Placenta for Nanosized Materials. Environ. Health Perspect. 2010, 118, 432-436.

39. Myllynen, P. K.; Loughran, M. J.; Howard, C. V.; Sormunen, R.; Walsh, A. A.; Vähäkangas, K. H. Kinetics of Gold Nanoparticles in the Human Placenta. Reprod. Toxicol. 2008, 26, 130-137.

40. Steel, C. D.; Stephens, A. L.; Hahto, S. M.; Singletary, S. J.; Ciavarra, R. P. Comparison of the Lateral Tail Vein and the Retro-Orbital Venous Sinus as Routes of Intravenous Drug Delivery in a Transgenic Mouse Model. Lab. Anim. (NY) 2008, 37, 26-32.

41. Whittaker, P.; Przyklenk, K. Fibrin Architecture in Clots: a Quantitative Polarized Light Microscopy Analysis. Blood Cells Mol. Dis. 2009, 42, 51-56.

42. Wörle-Knirsch, J. M.; Pulskamp, K.; Krug, H. F. Oops They Did it Again! Carbon Nanotubes Hoax Scientists in Viability Assays. Nano Lett. 2006, 6, 1261-1268.

43. LeBel, C. P.; Ischiropoulos, H.; Bondy, S. C. Evaluation of the Probe $2^{\prime}, 7^{\prime}$-Dichlorofluorescin as an Indicator of Reactive Oxygen Species Formation and Oxidative Stress. Chem. Res. Toxicol. 1992, 5, 227-231. 